\title{
Similarities and differences between mechanisms of organ and tissue growth regulation
}

\author{
BY R. J. GOSS \\ Division of Biology and Medicine, Brown University, Providence, Rhode Island 02912, USA
}

There are hundreds of different kinds of cells in the body, the numbers and sizes of which have to be carefully regulated if the disproportions of cancer and dystrophy are to be avoided. Since there must be as many regulatory mechanisms as there are cell types to be controlled, the question may be asked to what extent these mechanisms are alike or different?

One prefers to think, along with William of Occam, that in the economy of nature processes are not multiplied beyond necessity. Indeed, it would be convenient for the student of development if the similarities between the multifarious mechanisms of growth regulation outnumbered their differences. Were this to be the case, it might be possible to identify fundamental principles in those examples which have been more thoroughly explored, and then apply them to other systems the way a secret code can be used to decipher cryptograms. What is the probability that this might be feasible?

Natural processes tend to be conservative. So it is that organisms are more alike than different despite the superficial diversity that prevails in the organic world. At the molecular level, for example, they use the same amino acids, possess the same carbohydrates, fats and proteins, and catalyse their reactions with much the same complement of enzymes. Virtually all cells utilize comparable metabolic processes and are endowed with similar populations of organelles. Mechanisms of inheritance and reproduction are very much alike in all organisms in spite of their intriguing range of variations. Indeed, the very process of evolution itself has remained basically unchanged since life began.

Thus, the physiological and developmental processes that have evolved share much in common simply because innovations arose by modifying previously existing ones. As Jacob (1977) has so aptly noted, evolution is a matter of 'tinkering', not engineering, because it depends on the mutation of pre-existing genes, not the creation of new ones. This is consistent with the fact that life has of course evolved without benefit of design.

Applied to mechanisms of growth, the previously mentioned principles might predict that diverse organs and tissues in the body would have utilized many of the same basic processes. Not only do different organs and tissues differentiate from the same embryonic cells, but they have also evolved from common ancestors. It is no surprise, therefore, that hyperplasia and hypertrophy, for example, are ubiquitous attributes of growth, although their respective distributions vary among different tissues. Similarly, one might predict a high probability that the regulatory mechanisms by which growth is controlled would share much in common. How much they share, and how much they do not, are important considerations in our quest for fundamental principles of development. In the following account, therefore, a picture of growth will be painted with broad strokes of the brush in an attempt to uncover generalizations useful in helping us to understand how control mechanisms may have evolved. 


\section{HOW GROWTH CONTROLS ARE ALIKE}

Similarities abound when different organs and tissues are compared. This is particularly evident in the rclationship between growth and function. Increased physiological activity tends to promote enlargement, while disuse is followed by atrophy. Although each cell type may be assigned a different function and, therefore, be subject to different physiological conditions by which its numbers are determined, the general theme of control by functional demand nevertheless encompasses a wide variety of tissues. By deduction from such an hypothesis, it might be predicted that altering the known functions of as yet untested organs could turn their growth on or off accordingly.

It follows that the more specialized the tissue the more amenable it should be to having its growth regulation deciphered. A case in point is the erythrocyte, perhaps the most specialized cell in the body because of its unique function and structure. Erythrocytes are further simplified by the fact that they have no histological architecture. Not surprisingly, their numbers are correlated with the availability of, and need for, oxygen. Although the erythrocyte count is ordinarily fairly constant, there is no fixed size to their population. Vertebrates are endowed with the capacity to adjust their erythrocyte numbers according to supply and demand relationships between the body's tissues and atmospheric $\mathrm{O}_{2}$. Erythrocyte counts, therefore, are no more absolute than is the boiling point of water. They fluctuate with altitude and physiological requirements, thereby exercising the ideal adaptability of cell number to functional demands.

It is interesting to note how erythrocytes are not controlled. What does not happen is that erythropoiesis is controlled by the direct effects of $\mathrm{O}_{2}$ on erythrocyte precursors in the marrow or spleen. Instead, it is mediated by a hormone, erythropoietin, secreted by cells in the kidneys which are themselves sensitive to $\mathrm{O}_{2}$ availability with respect to need. Why this indirect mechanism occurs may date back to the evolutionary origins of erythropoiesis. In fishes, which lack marrow cavities, erythrocyte production goes on mostly in the kidneys. Initially, the control mechanism may have been anatomically close to the responding cells. When the latter moved to the marrow once bones became hollow, the former apparently stayed behind in the kidneys. Thus, erythrocyte precursors are stimulated by a growth factor (erythropoietin), but the growth factor itself is secreted in response to physiological need.

A similar situation prevails in other endocrinc relationships. Various trophic hormones. secreted when physiological demands are elevated, not only stimulate their target organs to greater functional activity, but also trigger cell hypertrophy and hyperplasia. Again, growth reactions are closely bound up with functional demands, in these cases indirectly mediated by third parties.

Although growth factors in the form of hormones cited previously may commonly be involved in growth regulation, this is not always the case. Other organs sometimes react directly to the physiological demands to which they are adapted. In the case of the parathyroid gland, for example, parathyroid hormone is secreted whenever serum calcium levels are low, there being no known trophic hormone involved. Calcitonin secretion likewise occurs in direct response to elevated serum $\mathrm{Ca}$. Comparable responses are seen in the islets of Langerhans and the zona glomerulosa of the adrenal cortex, where both growth and heightened hormone production occur in direct response to levels of blood sugar or sodium respectively.

Skeletal growth, at least in width, is also subject to direct regulation. Experiments have shown that piezoelectric forces, generated locally under mechanical pressure, 
mediate the resorption and deposition of minerals in the process of bone remodelling. Similarly, skeletal muscle grows in response to exercise or atrophies from disuse. Although denervation atrophy of muscle is a familiar phenomenon, the nerves themselves do not produce a trophic substance to promote enlargement of the muscle fibres they innervate. Rather, it is mechanical tension that causes muscle to hypertrophy. Even in the absence of a nerve supply, muscle fibres will differentiate in tissue culture (Konigsberg et al. 1975), denervated infant muscles will continue to grow as the skeletal parts to which they are attached elongate (Stewart, 1968), and passively stretched or exercised muscles will fail to atrophy after denervation (Stewart et al. 1972). Thus, no growth-promoting substance per se seems necessary for muscle growth.

Not every example of growth is so easily explained as are the aforementioned cases. Some of the most important organs are notoriously resistant to analysis. One reason for this is that they may be too physiologically complex. Instead of having but a single function, they have many. The liver, for example, carries out numerous activities that may conservatively be numbered in the dozens. Its famous capacity for regeneration, or rather compensatory hyperplasia, following partial hepatectomy, has successfully eluded attempts to pinpoint a single 'hepatotrophic' agent responsible for stimulating the burst of mitotic activity that takes place 1-2 d after subtotal resection. On the other hand, when individual functions of intact livers are stimulated (as when specific toxins, such as phenobarbital or carcinogens, are administered for detoxification), modest degrees of hyperplasia ensue (Augenlicht \& Argyris, 1975). Such responses are dose-dependent as well as additive (Argyris \& Heinemann, 1975), implying that the heroic extent of hyperplasia stimulated by partial hepatectomy may in fact represent the sum of many stimuli, one for each of the interrupted functions carried out by the liver. Such an interpretation would make sense in the economy of the body, for it would ensure that each organ would grow to a proportion commensurate with the physiological needs to be met.

It should not be accepted without question, however, that each organ and tissue represents a specific fraction of the body mass. While this interpretation may be a tautology, it is nevertheless important to ask why these proportions are as they are. In contemplating this question, it becomes apparent that the proportions of various organs in relation to each other and to the body as a whole are not necessarily fixed. As allometric studies have shown, they vary with age and size, and are influenced by development of the individual as well as the evolution of the species. They are also profoundly affected by physiological conditions, including pathological states. As Brody (1945) so succinctly put it, 'the organism changes geometrically so as to remain the same physiologically'.

\section{HOW GROWTH CONTROLS DIFFER}

One of the most basic differences between regulatory mechanisms depends on the distinction between somatic and visceral organs and tissues. Somatic tissues are those concerned with the more mechanical functions of the body. They include muscle, heart, blood vessels, bone, skin and connective tissues. Their growth is controlled by local influences rather than systemically distributed agents. When their masses are reduced, compensatory growth is, therefore, a local response, not one expressed by homologous tissues everywhere in the body. Visceral organs, on the other hand, are for the most part 
located in the body cavities enveloped by somatic tissues. Their functions tend to be more chemical than physical, involving the secretion of various products as well as the biochemical processing of materials. These organs are represented by blood cells, as well as endocrine and certain exocrine glands, including the kidneys and lungs. Their growth is regulated by bodywide influences such that all homologous cells are affected regardless of location in the body. To quote Abercrombie (1957), "either the stimulant is systemically distributed by the blood stream, the response being localized only because the sensitive cells are localized; or the stimulant is predominantly local, the responding cells being not so much those sensitive to as those exposed to stimulation.

Another difference is how the growth of various organs and tissues is controlled relates to how circuitous the feedback loop is between the demand for growth and the stimulus by which it is triggered. In some cases, the cells respond directly to the needs, in others they may react to other organs which in turn monitor growth. Endocrine glands are classic examples of such arrangements.

By analogy with moving vehicles, one may ask if growth is controlled by accelerators, brakes, or both? Some hypotheses favour stimulators, others inhibitors, and still others a combination of the two. This brings up the question of whether or not cells tend normally not to grow unless stimulated by some outside agency, or if they are naturally inclined to enlarge or proliferate unless prevented from doing so by exogenous inhibitors. In other words, is growth the absence of inhibition and atrophy the lack of stimulation, or does growth depend on stimulation and atrophy on inhibition? Either way, one wonders if such agents are produced by the affected organs themselves, or from outside sources, and in the former case if they are intra- or extracellular.

Growth is a two-way street. In fact, it is a street on a hillside where some processes promote increases in mass, others decreases. The question is whether or not this metaphor includes a gravitational field. It is not difficult to imagine that positive growth might be an active process requiring some kind of stimulus to initiate and sustain it. It is not so obvious, however, that atrophy (i.e. negative growth) might be a passive process. In the ongoing physiological turnover of the body that is so diagnostic of life itself. the 'normal' sizes of organs and tissues represent resultants of whatever processes are responsible for growth and atrophy. Clearly, the negative side of turnover deserves more attention than it has thus far received if we are to understand both directions of growth.

As will be elaborated below, the hypothesis that growth is controlled by functional demands is very appealing. Otherwise, one would have to resort to explanations that predict that tissue mass, not function, is the key to understanding growth. This dilemma represents a version of the age-old controversy between form and function. It is undeniable that there can be no function without some sort of form, but it is not so obvious that morphology can exist without function. Perhaps the only way out of this dilemma is to concede that growth and development are themselves functional processes.

However prevalent the importance of functional demand may be in controlling the growth and size of organs, the fact remains that there are important exceptions to this rule. Not the least of these is the growth that occurs during embryonic development, growth that clearly precedes functional activities that are destined to commence only at later stages of maturation. Such growth is sui generis, and its explanation remains to be found. Even in the adult, certain kinds of growth appear to be spontaneous. Epidermal appendages, for example, typically exhibit cyclic episodes of growth as when hairs and feathers are moulted and replaced, or when teeth, horns and hooves elongate indepen- 
dent of use. The mathematical precision with which teeth are shed and regrown, especially in lower vertebrates (Berkovitz, 1977; Cooper, 1966), is another example of how the growth of some structures anticipates function, not the other way around. Such cases as these are exaggerated versions of the physiological regeneration that goes on in virtually all organs at other levels of organization. They remind us of our profound ignorance about how turnover in either direction is controlled.

One might predict that if growth were subject exclusively to functional demands, that total disuse ought to lead to the complete disappearance of the unemployed organ. Though disuse inevitably brings about atrophy, it seldom if ever results in total obliteration. Organs and tissues may dwindle to a fraction of their former masses, but a remnant always persists, even for years. Denervated muscles may persist as atrophied relics (Gutmann \& Zelená, 1962), and bypassed urinary bladders plateau at about one-third of their original sizes (Goss \& Singleton, 1971). Pcrhaps this reservation has evolved as a means by which restoration might be achieved should function ever return, for once an organ disappears completely there would be no source from which it might later regenerate. Even so, this argues against the role of functional demand in explaining the growth and maintenance of all tissues.

Finally, one must consider the fact that there are several times as much of most organs as are needed for bare survival. If their growth were regulated by functional demands, how does one explain the redundancy that is so commonly exhibited in the masses of almost all tissues and organs. Animals can survive the loss of up to three-quarters of some of their most vital organs (e.g. kidneys, lungs, liver). Yet in each case remnants are stimulated to undergo compensatory growth, albeit concomitant with compensatory function. It is not difficult to appreciate how this margin of safety evolved, but it is challenging to explain the proximate mechanisms by which such reactions occur.

\section{CONCLUSION}

The question of whether growth-regulating mechanisms in various organs or tissues are more alike or different yields an ambiguous reply. There are many similarities and many differences. Similarities include the universal distribution of hypertrophy and hyperplasia, the ubiquity of physiological turnover, and the prevalence of use and disuse in promoting positive $v$. negative growth respectively. Yet the diversity of options exhibited by mechanisms of growth control caution against premature gencralizations. Differences between somatic and visceral organs, direct and indirect feedback controls, the role of stimulators $v$. inhibitors, and apparent exceptions to the role of functional demand in controlling organ size, all testify to the versatility with which organs and tissues have evolved differing mechanisms of growth regulation. Unhappily, such differences do not facilitate extrapolation from well-understood systems to less-explored ones. The task of the future, therefore, may be more difficult than one might have hoped. But if ignorance, like virtue, is diminished by becoming aware of it, then the admission of how little we know will itself be a step in the right direction.

\section{REFERENCES}

Abercrombie, M. (1957). Localized formation of new tissue in an adult mammal. Symposia of the Society for Experimental Biology 11, 235-254. 
Argyris, T. S. \& Heinemann, R. (1975). Ribosome accumulation in 3-methylcholanthrene-induced liver growth in adult male rats. Experimental and Molecular Pathology 22, 335-341.

Augenlicht, L. H. \& Argyris, T. S. (1975). Stimulation of immature male rat liver growth by phenobarbital and 3-methylcholanthrene. Experimental and Molecular Pathology 22, 1-10.

Berkovitz, B. K. B. (1977). The order of tooth development and eruption in the rainbow trout (Salmo gairdneri). Journal of Experimental Zoology 201, 221-225.

Brody, S. (1945). Bioenergetics and Growth. Princeton: Van Nostrand-Reinhold.

Cooper, J. S. (1966). Tooth replacement in amphibians and reptiles. British Journal of Herpetology 3, $214-218$.

Goss, R. J. \& Singleton. S. D. (1971). Disuse atrophy of the urinary bladder after bilateral nephrectomy. Proceedings of the Society of Experimental Biology and Medicine 138, 861-864.

Gutmann, E. \& Zelená, J. (1962). Morphological changes in the denervated muscle. In The Denervated Muscle, pp. 57-102 [E. Gutmann, editor]. Prague: Publishing House of the Czechoslovak Academy of Sciences.

Jacob, F. (1977). Evolution and tinkering. Science 196, 1161-1166.

Konigsberg, U. R., Lipton, B. H. \& Konigsberg, I. R. (1975). The regenerative response of single mature muscle fibres isolated in vitro. Developmental Biology 45, 260-275.

Stewart, D. M. (1968). Effect of age on the response of four muscles of the rat to denervation. American Journal of Physiology 214, 1139-1146.

Stewart, D. M., Sola. O. M. \& Martin, A. W. (1972). Hypertrophy as a response to denervation in skeletal muscle. Zeitschrift für Vergleichende Physiologie 76, 146-167. 